\title{
RIEMANN PROBLEM FOR HYPERBOLIC SYSTEMS OF CONSERVATION LAWS WITH NO CLASSICAL WAVE SOLUTIONS
}

BY

\author{
DECHUN TAN
}

Institute of Applied Mathematics, Academia Sinica, Beijing, China

1. Introduction. The theory of nonlinear hyperbolic systems of conservation laws usually assumes the systems to be strictly hyperbolic with genuinely nonlinear or linearly degenerate characteristic fields. General results of existence of entropy weak solutions to these systems are known only for initial data with small total variation (see Glimm [6], Lax [9], and Liu [10]). A natural question is whether these results remain true for a nonstrictly hyperbolic system, or even for strictly hyperbolic systems for initial data with large total variation. Recently, Keyfitz and Kranzer [7] considered the Riemann problem for

$$
\left\{\begin{array}{l}
u_{t}+\left(u^{2}-v\right)_{x}=0, \\
v_{t}+\left(\frac{1}{3} u^{3}-u\right)_{x}=0,
\end{array}\right.
$$

which is a genuinely nonlinear hyperbolic system. They found that for large $\left|U_{l}-U_{r}\right|$, the Riemann problem may admit no classical wave solution (which consists of contact discontinuities, shock, and/or rarefaction waves). Korchinski [8] studied the Riemann problem for

$$
\left\{\begin{array}{l}
u_{t}+\left(u^{2}\right)_{x}=0 \\
v_{t}+(u v)_{x}=0
\end{array}\right.
$$

which is a nonstrictly hyperbolic system. For some initial states $U_{l}$ and $U_{r}$, the Riemann problem admits no classical wave solutions even if $\left|U_{l}-U_{r}\right|$ is small enough. In [8] the Riemann problem is solved by introducing a new elementary wave-singular shock (we call it $\delta$-shock). Tan et al. [11] proved that the Riemann solution in [8] can be obtained by viscosity dissipation through

$$
\begin{aligned}
& \left\{\begin{array}{l}
u_{t}+\left(u^{2}\right)_{x}=\varepsilon t u_{x x}, \\
v_{t}+(u v)_{x}=0,
\end{array}\right. \\
& \left\{\begin{array}{l}
u_{t}+\left(u^{2}\right)_{x}=\varepsilon u_{x x}, \\
v_{t}+(u v)_{x}=0 .
\end{array}\right.
\end{aligned}
$$

Another result for nonstrictly hyperbolic systems is obtained in Le Floch [4].

Received October 21, 1991.

1991 Mathematics Subject Classification. Primary 35L65, 35L67, 76L05.

(C)1993 Brown University 
In the first part of this paper we consider the Riemann problem for

$$
\left\{\begin{array}{l}
u_{t}+\left(u^{2}\right)_{x}=0, \\
v_{t}+((2 u+1) v)_{x}=0,
\end{array}\right.
$$

which is a strictly hyperbolic system with linearly degenerate characteristic fields. The same occurrence of the nonexistence in [7] is the case for this system. Analogous to [8], we first solve the Riemann problem by introducing $\delta$-waves along which $v$ is a Dirac $\delta$-function $c(x, t) \cdot \delta(x-\sigma t)$ and $u$ is a step function. $u \cdot v$ is a Borel measure as defined in [11]. Then we prove that these solutions are just the limit of $U_{1}^{\varepsilon}(x, t)=\left(u^{\varepsilon}(x, t), v^{\varepsilon}(x, t)\right)$ as $\varepsilon \rightarrow 0^{+}$. Here $U_{1}^{\varepsilon}(x, t)$ is the unique self-similar solution for the corresponding Riemann problem of

$$
\left\{\begin{array}{l}
u_{t}+\left(u^{2}\right)_{x}=\varepsilon t u_{x x}, \\
v_{t}+((2 u+1) v)_{z}=0 .
\end{array}\right.
$$

In the second part of this paper, we return to (1.2) and prove that the Riemann solution in [8] is the limit of $U_{2}^{\varepsilon}(x, t)$ as $\varepsilon \rightarrow 0^{+}$. Here $U_{2}^{\varepsilon}(x, t)$ is the self-similar solution of

$$
\left\{\begin{array}{l}
u_{t}+\left(u^{2}\right)_{x}=\varepsilon t u_{x x}, \\
v_{t}+(u v)_{x}=\varepsilon t v_{x x} .
\end{array}\right.
$$

It is anticipated that the same result is true for (1.5).

We especially emphasize that the Riemann solutions of (1.2) (resp. (1.5)), which only involve classical waves, can be obtained in the same way as in Le Floch [3,4] but those involving delta-shock cannot.

2. Riemann solutions for (1.5). Under the self-similar transformation $\xi=x / t$, (1.5) becomes

$$
\left\{\begin{array}{l}
-\xi u_{\xi}-\left(u^{2}\right)_{\xi}=0, \\
-\xi v_{\xi}+((2 u+1) v)_{\xi}=0,
\end{array}\right.
$$

and the initial condition is written as

$$
(u, v)( \pm \infty)=\left(u^{ \pm}, v^{ \pm}\right) .
$$

If in a domain the solution is smooth but not constant, then from (2.1) we get

$$
\left\{\begin{array}{l}
\xi=2 u, \\
\frac{v}{v_{0}}=\frac{e^{u_{0}}}{e^{u}} .
\end{array}\right.
$$

We call the solution in this domain a rarefaction wave, denoted by $R$.

Assume $\xi=\sigma$ is a nonsingular discontinuity point. By the Rankine-Hugoniot condition

$$
\left\{\begin{array}{l}
\sigma[u]=\left[u^{2}\right], \\
\sigma[v]=[(2 u+1) v],
\end{array}\right.
$$

we get

$$
\sigma_{2}=2 u_{l}+1=2 u_{r}+1
$$




$$
\left\{\begin{array}{l}
\sigma_{1}=u_{r}+u_{l}, \\
\frac{v_{r}}{v_{l}}=-\frac{u_{r}-u_{l}-1}{u_{r}-u_{l}+1},
\end{array}\right.
$$

where $[u]=u_{l}-u_{r},[v]=v_{l}-v_{r}$.

The discontinuity satisfying (2.5.1) is called a contact discontinuity, denoted by $J$. The discontinuity satisfying (2.5.2) as well as the "3-incoming, 1-outgoing" shock condition is called a shock wave, denoted by $S$. We call the discontinuity satisfying $\sigma=u_{r}+u_{l}$ as well as the "4-incoming" $\delta$-entropy condition a delta-shock, denoted by $\delta$. Here the concept "3-incoming, 1-outgoing" and "4-incoming" are similar to those in [11]. We write them in inequality form as (2.6) and (2.7) respectively:

$$
\begin{gathered}
\lambda_{1 r}<\sigma_{1}<\lambda_{1 l}<\lambda_{2 l}, \quad \sigma_{1}<\lambda_{2 r}, \\
\lambda_{1 r}<\lambda_{2 r}<\sigma_{1}<\lambda_{1 l}<\lambda_{2 l},
\end{gathered}
$$

where $\lambda_{1}=2 u<\lambda_{2}=2 u+1$.

Having defined these elementary waves, we can construct the Riemann solutions with analysis in the phase plane. The wave curve starting from $\left(u^{-}, v^{-}\right)$is illustrated in Fig. 2.1.

For $u^{-}-1<u^{+}<u^{-}$, the solution is $S+J$; see Fig. 2.2 on p. 768 .

For $u^{+}<u^{-}-1$, the solution is delta-shock; see Fig. 2.3 on p. 768 .

For $u^{+}>u^{-}$, the solution is $R+J$; see Fig. 2.4 on p. 768 .

Remark. Suppose $u^{+}=u^{-}-1+\varepsilon$ with $\varepsilon>0, v^{+}$fixed. As we have seen in Fig. 2.2, the Riemann problem admits an $S+J$ solution. Denote the $v$-component by $v_{\varepsilon}(x, t)$. Here

$$
\begin{gathered}
u^{*}=u^{+}, \quad \sigma_{1}=u^{-}+u^{*}, \quad \sigma_{2}=2 u^{+}+1, \\
v^{*}=-\frac{v^{-}\left(u^{+}-u^{-}-1\right)}{u^{+}-u^{-}+1} .
\end{gathered}
$$

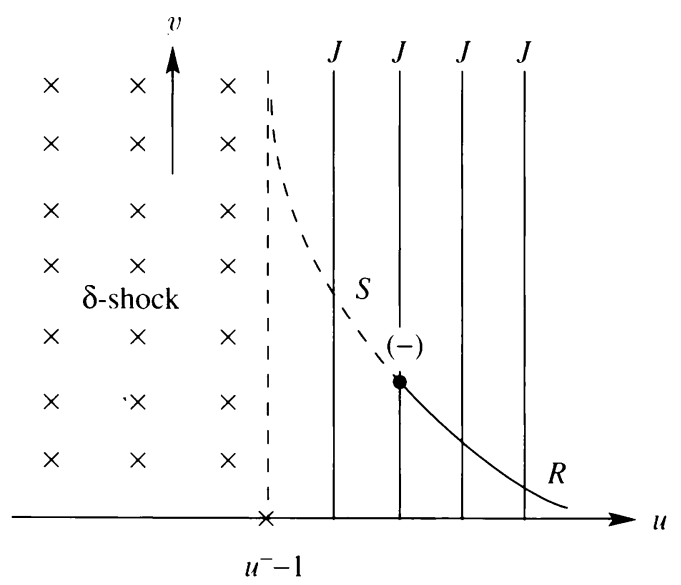

FIG. 2.1 


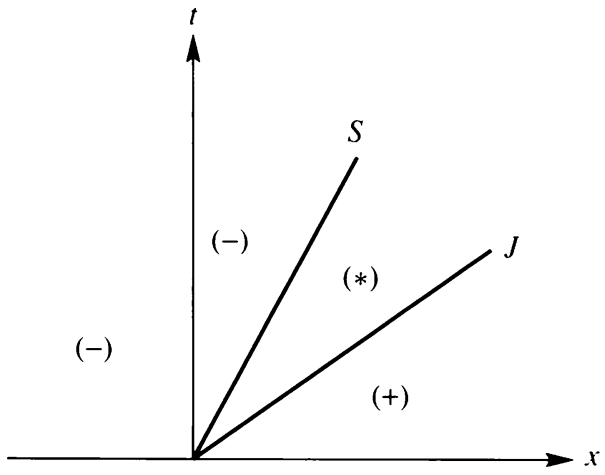

FIG. 2.2.

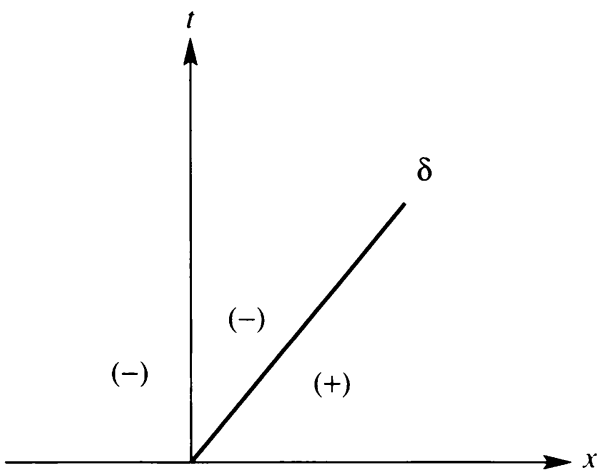

FIG. 2.3.

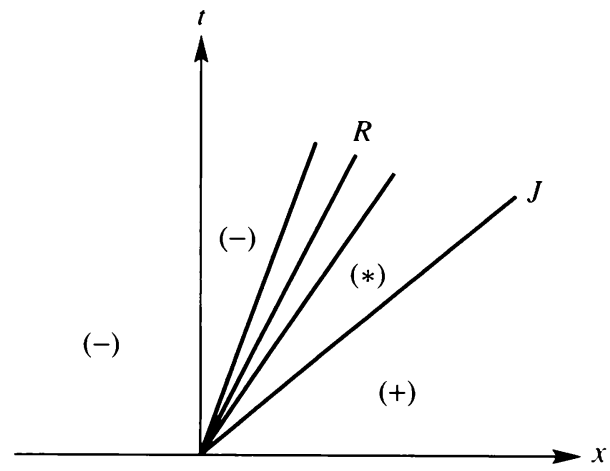

Fig. 2.4.

From the Rankine-Hugoniot condition

$$
\left\{\begin{array}{l}
\sigma_{2}\left(v^{+}-v^{*}\right)=\left(2 u^{+}+1\right) v^{+}-\left(2 u^{*}+1\right) v^{*}, \\
\sigma_{1}\left(v^{*}-v^{-}\right)=\left(2 u^{*}+1\right) v^{*}-\left(2 u^{-}+1\right) v^{-},
\end{array}\right.
$$

we get

$$
\sigma_{2} v^{+}-\sigma_{1} v^{-}-\left(\sigma_{2}-\sigma_{1}\right) v^{*}=\left(2 u^{+}+1\right) v^{+}-\left(2 u^{-}+1\right) v^{-} .
$$

When $\varepsilon \rightarrow 0^{+}$, we find $\sigma_{1}, \sigma_{2} \rightarrow 2 u^{-}-1$. By a simple calculation, we obtain 


$$
\lim _{\varepsilon \rightarrow 0^{+}}\left(\sigma_{2}-\sigma_{1}\right) v^{*}=2 v^{-},
$$

which means that when $u^{+}=u^{-}-1$ the solution we constructed which involves a delta-shock is the limit of the classical wave solution as $\varepsilon \rightarrow 0^{+}$. The limit solution does not satisfy the Rankine-Hugoniot condition.

3. Existence and limit of the solutions for (1.6). Consider the Riemann problem of (1.6). Performing the self-similar transformation $\xi=x / t$, we get

$$
\left\{\begin{array}{l}
(2 u-\xi) u_{\xi}=\varepsilon u_{\xi \xi} \\
-\xi v_{\xi}+((2 u+1) v)_{\xi}=0
\end{array}\right.
$$

The initial condition becomes

First, we solve

$$
(u, v)( \pm \infty)=\left(u^{ \pm}, v^{ \pm}\right) .
$$

$$
\left\{\begin{array}{l}
(2 u-\xi) u_{\xi}=\varepsilon u_{\xi \xi} \\
u( \pm \infty)=u^{ \pm}
\end{array}\right.
$$

One can easily verify the existence, smoothness, uniqueness, and monotonicity of the solution for (3.3). For convenience, we assume $u^{+}<u^{-}$, so $u^{\varepsilon}(\xi)$ is strictly decreasing.

Substituting $u^{\varepsilon}(\xi)$ in the second equation of (3.1), we get

$$
\left\{\begin{array}{l}
-\xi v_{\xi}+\left(\left(2 u^{\varepsilon}+1\right) v\right)_{\xi}=0, \\
v( \pm \infty)=v^{ \pm}
\end{array}\right.
$$

which is a two-point boundary value problem for the first-order ordinary equation. $\xi=\xi_{\alpha}^{\varepsilon}: \xi_{\alpha}^{\varepsilon}=2 u^{\varepsilon}\left(\xi_{\alpha}^{\varepsilon}\right)+1$ is the unique singular point of (3.4.1).

As in [11], we solve (3.4) in $\left(-\infty, \xi_{\alpha}^{\varepsilon}\right)$ and $\left(\xi_{\alpha}^{\varepsilon},+\infty\right)$ and the solutions are $v_{1}^{\varepsilon}(\xi)$ and $v_{2}^{\varepsilon}(\xi)$, respectively.

$$
v_{1}^{\varepsilon}(-\infty)=v^{-}, \quad v_{2}^{\varepsilon}(+\infty)=v^{+},
$$

$v_{1}^{\varepsilon}(\xi)$ and $v_{2}^{\varepsilon}(\xi)$ are monotone functions of $\xi$, and

$$
\begin{gathered}
\lim _{\xi \rightarrow \xi_{\alpha}^{\varepsilon}-0}\left|\int_{\xi_{1}}^{\xi} v_{1}^{\varepsilon}(\xi) d \xi\right|=A_{1}<\infty, \\
\lim _{\xi \rightarrow \xi_{\alpha}^{\varepsilon}+0}\left|\int_{\xi_{2}}^{\xi} v_{2}^{\varepsilon}(\xi) d \xi\right|=A_{2}<\infty, \\
\lim _{\xi \rightarrow \xi_{\alpha}^{\varepsilon}-0}\left(2 u^{\varepsilon}(\xi)+1-\xi\right) v_{1}^{\varepsilon}(\xi)=0, \\
\lim _{\xi \rightarrow \xi_{\alpha}^{\varepsilon}+0}\left(2 u^{\varepsilon}(\xi)+1-\xi\right) v_{2}^{\varepsilon}(\xi)=0 .
\end{gathered}
$$

Set

$$
v^{\varepsilon}(\xi)= \begin{cases}v_{1}^{\varepsilon}(\xi), & \xi<\xi_{\alpha}^{\varepsilon}, \\ v_{2}^{\varepsilon}(\xi), & \xi>\xi_{\alpha}^{\varepsilon} .\end{cases}
$$

We can easily prove that $v^{\varepsilon}(\xi)$ is a weak solution of (3.4), i.e., $v^{\varepsilon}(\xi) \in L_{\text {loc }}^{1}(R)$ and $\forall \phi \in C_{0}^{\infty}(R)$ :

$$
-\int_{R}(2 u+1-\xi) v^{\varepsilon} \phi^{\prime} d \xi+\int_{R} v^{\varepsilon} \phi d \xi=0
$$


Furthermore, we have that

$$
\begin{aligned}
& -\int_{-\infty}^{\xi_{\alpha}^{\varepsilon}}(2 u+1-\xi) v^{\varepsilon} \phi^{\prime} d \xi+\int_{-\infty}^{\xi_{\alpha}^{\varepsilon}} v^{\varepsilon} \phi d \xi=0, \\
& -\int_{\xi_{\alpha}^{\varepsilon}}^{+\infty}(2 u+1-\xi) v^{\varepsilon} \phi^{\prime} d \xi+\int_{\xi_{\alpha}^{\varepsilon}}^{+\infty} v^{\varepsilon} \phi d \xi=0
\end{aligned}
$$

hold for any $\phi \in C_{0}^{\infty}(R)$.

To investigate the limit behavior of the solution for $\varepsilon \rightarrow 0^{+}$, we employ

Lemma 3.1. Let $\xi_{\beta}^{\varepsilon}$ denote the unique point satisfying $\xi_{\beta}^{\varepsilon}=2 u^{\varepsilon}\left(\xi_{\beta}^{\varepsilon}\right)$, and let $\xi_{\beta}$ be a limit point of $\xi_{\beta}^{\varepsilon}$ for $\varepsilon \rightarrow 0^{+}$. Then for any $\delta>0$,

$$
\begin{array}{ll}
\lim _{\varepsilon \rightarrow 0^{+}} u_{\xi}^{\varepsilon}(\xi)=0 & \text { for }\left|\xi-\xi_{\beta}\right|>\delta, \\
\lim _{\varepsilon \rightarrow 0^{+}} u^{\varepsilon}(\xi)=u^{+} & \text {for } \xi>\xi_{\beta}+\delta, \\
\lim _{\varepsilon \rightarrow 0^{+}} u^{\varepsilon}(\xi)=u^{-} & \text {for } \xi<\xi_{\beta}-\delta
\end{array}
$$

hold uniformly in the above intervals.

LeMMA 3.2. Let $\xi_{\alpha}^{\varepsilon}: \xi_{\alpha}^{\varepsilon}=2 u^{\varepsilon}\left(\xi_{\alpha}^{\varepsilon}\right)+1$. Assume $\xi_{\alpha}$ is a limit point of $\xi_{\alpha}^{\varepsilon}$ for $\varepsilon \rightarrow 0^{+}$. Then

(i) $u^{-}>u^{+}>u^{-}-1$ implies $\xi_{\alpha}=2 u^{+}+1$.

(ii) $u^{-}-1>u^{+}$implies $\xi_{\alpha}=\xi_{\beta}=\sigma_{\delta}=u^{+}+u^{-}$.

Lemma 3.3. For any $\delta>0$, we have

$\lim _{\varepsilon \rightarrow 0+} v^{\varepsilon}(\xi)=v^{+}$uniformly for $\xi>\xi_{\alpha}+\delta$;

$\lim _{\varepsilon \rightarrow 0+} v^{\varepsilon}(\xi)=v^{*}$ uniformly for $\xi_{\beta}+\delta<\xi<\xi_{\alpha}-\delta$ (when $u^{+}>u^{-}-1$ );

$\lim _{\varepsilon \rightarrow 0+} v^{\varepsilon}(\xi)=v^{-}$uniformly for $\xi<\xi_{\beta}-\delta$.

The proofs are similar to those in [11]; so we omit them.

Based on Lemmas 3.1-3.3, we know that

(i) when $u^{-}>u^{*}>u^{-}-1, \xi_{\alpha}=2 u^{+}+1>\xi_{\beta}=u^{+}+u^{-}$, and we can obtain $\left|U^{\varepsilon}(\xi)\right| \leq M$ uniformly for $\xi<\xi_{\alpha}-\delta, \delta>0, M=M(\delta)$. So if we denote $U^{0}(\xi)=\lim _{\varepsilon \rightarrow 0^{+}} U^{\varepsilon}(\xi)$ a.e. and if $\bar{U}(\xi)$ is the Riemann solution for (1.6) constructed in Sec. 2, then $U^{0}(\xi)$ satisfies the Rankine-Hugoniot conditions at $\xi=\xi_{\beta}$, i.e.,

$$
v_{0}^{*}=-\frac{v^{-}\left(u^{+}-u^{-}-1\right)}{u^{+}-u^{-}+1} .
$$

That is, the Riemann solution involving $J$ and $S$ is the limit of the solution of (3.1), (3.2) as $\varepsilon \rightarrow 0^{+}$.

(ii) When $u^{-}-1>u^{+}, \xi_{\alpha}=\xi_{\beta}=\sigma=u^{+}+u^{-}$; so $U^{0}(\xi) \equiv \bar{U}(\xi)$ for $\xi \neq \sigma$. That is, the Riemann solution containing a delta-shock is also the limit of the solution of (3.1), (3.2) almost everywhere.

The interesting part is the limit behavior in the neighborhood of $\xi=\sigma=u^{+}+u^{-}$ as $\varepsilon \rightarrow 0^{+}$. To investigate it, we take $\phi \in C_{0}^{\infty}\left[\xi_{1}, \xi_{2}\right]\left(\xi_{1}<\sigma<\xi_{2}\right)$ such that 
$\phi(\xi) \equiv \phi(\sigma)$ for $\xi$ in a neighborhood $U$ of $\xi=\sigma$ (we call such a $\phi$ a sloping test function). When $0<\varepsilon<\varepsilon_{0}, \xi_{\alpha}^{\varepsilon} \in U$.

Returning to (3.10), by virtue of Lemmas 3.1-3.3, we immediately have

$$
\begin{gathered}
\lim _{\varepsilon \rightarrow 0^{+}} \int_{\xi_{1}}^{\xi_{0}^{\varepsilon}}\left(v^{\varepsilon}-H\left(\xi-\xi_{\alpha}^{\varepsilon}\right)\right) \phi(\xi) d \xi=-\left(\left(2 u^{-}+1\right) v^{-}-\sigma v^{-}\right) \phi(\sigma), \\
\lim _{\varepsilon \rightarrow 0^{+}} \int_{\xi_{\alpha}^{\varepsilon}}^{\xi_{2}}\left(v^{\varepsilon}-H\left(\xi-\xi_{\alpha}^{\varepsilon}\right)\right) \phi(\xi) d \xi=\left(\left(2 u^{+}+1\right) v^{+}-\sigma v^{+}\right) \phi(\sigma)
\end{gathered}
$$

for any sloping test function. Here

$$
H(x)= \begin{cases}v^{-}, & x<0 \\ v^{+}, & x>0\end{cases}
$$

By the monotonicity of $v_{1}^{\varepsilon}(\xi), v_{2}^{\varepsilon}(\xi)$, and by using the same approximation argument as in [11], we obtain

$$
\lim _{\varepsilon \rightarrow 0^{+}} \int_{\xi_{1}}^{\xi_{2}}\left(v^{\varepsilon}-H\left(\xi-\xi_{\alpha}^{\varepsilon}\right)\right) \phi(\xi) d \xi=([(2 u+1) v]-\sigma[v]) \phi(\sigma)
$$

for all $\phi \in C_{0}^{\infty}\left[\xi_{1}, \xi_{2}\right]$, which implies

THEOREM 3.4. Suppose $U^{\varepsilon}(\xi)$ is a solution of $(3.1),(3.2)$ and $u^{+}<u^{-}-1$. Then $v^{\varepsilon}(\xi)-H\left(\xi-\xi_{\alpha}^{\varepsilon}\right)$ converges weakly to $c \cdot \delta(\xi-\sigma)$ for $\varepsilon \rightarrow 0^{+}$, where $\delta$ is the Dirac $\delta$-function, and $c=[(2 u+1) v]-\sigma[v]$.

4. Existence and limit of the solutions for (1.7). In this section, we consider the Riemann problem

$$
\left\{\begin{array}{l}
u_{t}+\left(u^{2}\right)_{x}=\varepsilon t u_{x x}, \\
v_{t}+(u v)_{x}=\varepsilon t v_{x x}, \\
(u, v)(x, 0)=\left(u^{ \pm}, v^{ \pm}\right) \pm x>0
\end{array}\right.
$$

with $u^{-}>0>u^{+}$. Other cases can be solved by the method of Dafermos [1] and Dafermos and DiPerna [2] or as follows.

Under the self-similar transformation $\xi=x / t,(4.1)$ becomes

$$
\left\{\begin{array}{l}
(2 u-\xi) u_{\xi}=\varepsilon u_{\xi \xi}, \\
-\xi v_{\xi}+(u v)_{\xi}=\varepsilon v_{\xi \xi}, \\
(u, v)( \pm \infty)=\left(u^{ \pm}, v^{ \pm}\right) .
\end{array}\right.
$$

First,

$$
\left\{\begin{array}{l}
(2 u-\xi) u_{\xi}=\varepsilon u_{\xi \xi} \\
u( \pm \infty)=u^{ \pm}
\end{array}\right.
$$

is uniquely solvable. Denote the solution by $u^{\varepsilon}(\xi)$. We know that $u_{\xi}^{\varepsilon}(\xi)<0$ as $u^{-}>u^{+}$. Substituting it into the second equation of (4.2), we are led to

$$
\left\{\begin{array}{l}
-\xi v_{\xi}+\left(u^{\varepsilon} v\right)_{\xi}=\varepsilon v_{\xi \xi}, \\
v( \pm \infty)=v^{ \pm}
\end{array}\right.
$$


As we shall see, the solution of (4.4) admits no uniform bounds with respect to $\varepsilon>0$. So it is difficult to solve the problem in the same frame as in $[1,2]$. Fortunately $v=u^{\varepsilon}(\xi)$ is a nontrivial solution of (4.4.1). By the general theory of second-order linear ordinary differential equations, we immediately get that

$$
p(\xi)=u(\xi) \int_{\xi_{1}}^{\xi} u^{-2}(s) \exp \left(\int_{\xi_{1}}^{s} \frac{u-\tau}{\varepsilon} d \tau\right) d s
$$

is another solution of (4.4.1) which is linearly independent from $u^{\varepsilon}(\xi)$. Since $\xi=\xi_{0}^{\varepsilon}$, $u^{\varepsilon}\left(\xi_{0}^{\varepsilon}\right)=0$ is the singular point of $p(\xi)$, we construct the solution on $\left(-\infty, \xi_{0}^{\varepsilon}\right)$ and $\left(\xi_{0}^{\varepsilon},+\infty\right)$, respectively.

Let $\xi_{1}, \xi_{2}$ be such that $\xi_{1}<\xi_{0}^{\varepsilon}<\xi_{2}$. Then

$$
\begin{aligned}
& p_{1}(\xi)=u(\xi) \int_{\xi_{1}}^{\xi} u^{-2}(s) \exp \left(\int_{\xi_{1}}^{s} \frac{u-\tau}{\varepsilon} d \tau\right) d s, \\
& p_{2}(\xi)=u(\xi) \int_{\xi_{2}}^{\xi} u^{-2}(s) \exp \left(\int_{\xi_{2}}^{s} \frac{u-\tau}{\varepsilon} d \tau\right) d s
\end{aligned}
$$

are just the solutions on $\left(-\infty, \xi_{0}^{\varepsilon}\right)$ and $\left(\xi_{0}^{\varepsilon},+\infty\right)$.

Integrating $p_{i}(\xi)$ by parts, we obtain

$$
\begin{gathered}
p_{i}(\xi)=-\frac{1}{u^{\prime}(\xi)} \exp \left(\int_{\xi_{i}}^{\xi} \frac{u-\tau}{\varepsilon} d \tau\right)+\frac{u(\xi)}{u\left(\xi_{i}\right) u^{\prime}\left(\xi_{i}\right)}-u(\xi) \int_{\xi_{i}}^{\xi} \frac{1}{u^{\prime}(s)} \exp \left(\int_{\xi_{i}}^{s} \frac{u-\tau}{\varepsilon} d \tau\right) d s, \\
p_{i}^{\prime}(\xi)=\frac{u(\xi)}{u^{\prime}\left(\xi_{i}\right)}\left\{\frac{1}{u\left(\xi_{i}\right)}-\int_{\xi_{i}}^{\xi} \exp \left(\int_{\xi_{i}}^{s} \frac{-u}{\varepsilon} d \tau\right) d s\right\} .
\end{gathered}
$$

So

$$
\begin{gathered}
\left\{\begin{array}{l}
p_{i}\left(\xi_{0}^{\varepsilon}\right)=-\frac{1}{u^{\prime}\left(\xi_{0}^{\varepsilon}\right)} \cdot q_{i}\left(\xi_{0}^{\varepsilon}\right), \\
p_{i}^{\prime}\left(\xi_{0}^{\varepsilon}\right)=\frac{v^{\prime}\left(\xi_{0}^{\varepsilon}\right)}{u^{\prime}\left(\xi_{i}\right)} \cdot r_{i}\left(\xi_{0}^{\varepsilon}\right),
\end{array}\right. \\
q_{i}\left(\xi_{0}^{\varepsilon}\right)=\exp \left(\int_{\xi_{i}}^{\xi_{0}^{\varepsilon}} \frac{u-\tau}{\varepsilon} d \tau\right), \\
r_{i}\left(\xi_{0}^{\varepsilon}\right)=\frac{1}{u\left(\xi_{i}\right)}-\int_{\xi_{i}}^{\xi_{0}^{\varepsilon}} \exp \left(\int_{\xi_{i}}^{s} \frac{-u}{\varepsilon} d \tau\right) d s .
\end{gathered}
$$

Let

and

$$
\left\{\begin{array}{l}
v_{1}(\xi)=c_{1} u(\xi)+c_{2} p_{1}(\xi) \\
v_{2}(\xi)=c_{3} u(\xi)+c_{4} p_{2}(\xi)
\end{array}\right.
$$

$$
v(\xi)= \begin{cases}v_{1}(\xi), & \xi<\xi_{0}^{\varepsilon}, \\ v_{2}(\xi), & \xi>\xi_{0}^{\varepsilon} .\end{cases}
$$

To solve (4.4), it suffices to determine $c_{1}, c_{2}, c_{3}, c_{4}$, such that

$$
\left\{\begin{array}{l}
c_{1} u^{-}+c_{2} p_{1}(-\infty)=v^{-} \\
c_{3} u^{+}+c_{4} p_{2}(+\infty)=v^{+} \\
c_{2} p_{1}\left(\xi_{0}^{\varepsilon}\right)=c_{4} p_{2}\left(\xi_{0}^{\varepsilon}\right) \\
c_{1} u^{\prime}\left(\xi_{0}^{\varepsilon}\right)+c_{2} p_{1}^{\prime}\left(\xi_{0}^{\varepsilon}\right)=c_{3} u^{\prime}\left(\xi_{0}^{\varepsilon}\right)+c_{4} p_{2}^{\prime}\left(\xi_{0}^{\varepsilon}\right) .
\end{array}\right.
$$


The last two equations in (4.12) are needed to guarantee $v\left(\xi_{0}^{\varepsilon}+0\right)=v\left(\xi_{0}^{\varepsilon}-0\right)$ and $v^{\prime}\left(\xi_{0}^{\varepsilon}+0\right)=v^{\prime}\left(\xi_{0}^{\varepsilon}-0\right)$. By (4.7), we know that (4.12) is equivalent to

$$
A c=b,
$$

where $c=\left(c_{1}, c_{2}, c_{3}, c_{4}\right)^{\mathrm{T}}, b=\left(v^{-}, v^{+}, 0,0\right)^{\mathrm{T}}$,

$$
A=\left(\begin{array}{cccc}
u^{-} & p_{1} & 0 & 0 \\
0 & 0 & u^{+} & p_{2} \\
0 & q_{1} & 0 & -q_{2} \\
u_{2}^{\prime} & u_{2}^{\prime} r_{1} / u_{1}^{\prime} & -u_{2}^{\prime} & -r_{2}
\end{array}\right),
$$

where $p_{1}=p_{1}(-\infty), p_{2}=p_{2}(+\infty), q_{i}=q_{i}\left(\xi_{0}^{\varepsilon}\right)$, and $u_{i}^{\prime}=u^{\prime}\left(\xi_{i}\right), r_{i}=r_{i}\left(\xi_{0}^{\varepsilon}\right)$,

$$
\begin{aligned}
\operatorname{det} A & =\left|\begin{array}{cccc}
u^{-} & p_{1} & 0 & 0 \\
0 & 0 & u^{+} & p_{2} \\
0 & q_{1} & 0 & -q_{2} \\
u_{2}^{\prime} & u_{2}^{\prime} r_{1} / u_{1}^{\prime} & -u_{2}^{\prime} & -r_{2}
\end{array}\right| \\
& =u^{-}\left|\begin{array}{ccc}
0 & u^{+} & p_{2} \\
q_{1} & 0 & -q_{2} \\
u_{2}^{\prime} r_{1} / u_{1}^{\prime} & -u_{2}^{\prime} & -r_{2}
\end{array}\right|-u_{2}^{\prime}\left|\begin{array}{ccc}
p_{1} & 0 & 0 \\
0 & u^{+} & p_{2} \\
q_{1} & 0 & -q_{2}
\end{array}\right| \\
& =u^{-}\left[-u_{2}^{\prime} q_{1} p_{2}-u^{+} q_{2} u_{2}^{\prime} r_{1} / u_{1}^{\prime}+u^{+} q_{1} r_{2}\right]+u_{2}^{\prime} q_{2} u^{+} p_{1} .
\end{aligned}
$$

From (4.5), (4.6), as well as $u^{+}<0<u^{-}$, we know

$$
p_{1}=p_{1}(-\infty)<0, \quad p_{2}=p_{2}(+\infty)<0 .
$$

From (4.8), (4.9), we have

$$
\lim _{\varepsilon \rightarrow 0^{+}} r_{1}\left(\xi_{0}^{\varepsilon}\right)=-\infty, \quad \lim _{\varepsilon \rightarrow 0^{+}} r_{2}\left(\xi_{0}^{\varepsilon}\right)=+\infty .
$$

So we can choose $\xi_{1}^{\varepsilon}<\xi_{0}^{\varepsilon}, \xi_{\alpha}^{\varepsilon}<\xi_{2}^{\varepsilon}$ such that

$$
\begin{gathered}
\frac{u_{2}^{\prime}\left(\xi_{2}^{\varepsilon}\right)}{u_{1}^{\prime}\left(\xi_{1}^{\varepsilon}\right)}=a>0, \\
r_{1}\left(\xi_{0}^{\varepsilon}\right) \leq-a, \quad r_{2}\left(\xi_{0}^{\varepsilon}\right) \geq a, \\
\lim _{\varepsilon \rightarrow 0^{+}} \xi_{1}^{\varepsilon}=\lim _{\varepsilon \rightarrow 0^{+}} \xi_{2}^{\varepsilon}=u^{+}+u^{-},
\end{gathered}
$$

where $a$ is a proper positive constant independent of $\varepsilon>0$. Hence, $\operatorname{det} A<0$ which implies (4.12) is uniquely solvable for any $v^{+}, v^{-}$, i.e.,

THEOREM 4.1. For any $\varepsilon>0, u^{+}<0<u^{-}$as well as $v^{+}, v^{-} \in R^{1}$, there exist $\left(u^{\varepsilon}(\xi), v^{\varepsilon}(\xi)\right) \in C^{2}(R)$ such that

$$
\left\{\begin{array}{l}
-\xi u_{\xi}+\left(u^{2}\right)_{\xi}=\varepsilon u_{\xi \xi}, \\
-\xi v_{\xi}+(u v)_{\xi}=\varepsilon v_{\xi \xi}, \\
(u, v)( \pm \infty)=\left(u^{ \pm}, v^{ \pm}\right) .
\end{array}\right.
$$

From the above construction, we know $v\left(\xi_{0}^{\varepsilon}-0\right)=v\left(\xi_{0}^{\varepsilon}+0\right)$ and $v^{\prime}\left(\xi_{0}^{\varepsilon}-0\right)=$ $v^{\prime}\left(\xi_{0}^{\varepsilon}+0\right)$. By (4.4.1), we get $v^{\prime \prime}\left(\xi_{0}^{\varepsilon}-0\right)=v^{\prime \prime}\left(\xi_{0}^{\varepsilon}+0\right)$. 
Lemma 4.2. Assume $u^{+}<0<u^{-}$, and $\xi_{0}, \xi_{\alpha}, \xi_{\beta}$ are the limit points of $\xi_{0}^{\varepsilon}, \xi_{\alpha}^{\varepsilon}, \xi_{\beta}^{\varepsilon}$, respectively, for $\varepsilon \rightarrow 0^{+}$. Then

$$
\xi_{0}=\xi_{\alpha}=\xi_{\beta}=u^{+}+u^{-} .
$$

Here $\xi_{\alpha}^{\varepsilon}=u^{\varepsilon}\left(\xi_{\alpha}^{\varepsilon}\right), \xi_{\beta}^{\varepsilon}=2 u^{\varepsilon}\left(\xi_{\beta}^{\varepsilon}\right)$.

THeOREM 4.3. Assume $\left(u^{\varepsilon}(\xi), v^{\varepsilon}(\xi)\right)$ constructed in (4.11) is a solution of (4.18). Then for any $\delta>0$

$$
\begin{array}{ll}
\lim _{\varepsilon \rightarrow 0^{+}} v^{\varepsilon}(\xi)=v^{-} & \text {for } \xi<\xi_{0}-\delta, \\
\lim _{\varepsilon \rightarrow 0^{+}} v^{\varepsilon}(\xi)=v^{+} & \text {for } \xi>\xi_{0}+\delta
\end{array}
$$

hold uniformly in the above intervals.

Proof. For $\xi<\xi_{0}^{\varepsilon}$,

$$
v^{\varepsilon}(\xi)=v_{1}^{\varepsilon}(\xi)=c_{1} u^{\varepsilon}(\xi)+c_{2} p_{1}^{\varepsilon}(\xi) .
$$

To investigate the limit behavior of $v_{1}^{\varepsilon}(\xi)$, we first find

$$
\lim _{\varepsilon \rightarrow 0^{+}} c_{1} \text { and } \lim _{\varepsilon \rightarrow 0^{+}} c_{2} .
$$

Returning to (4.13), we have

$$
c_{2}=\frac{u_{2}^{\prime}\left(v^{-} u^{+}-v^{+} u^{-}\right)}{p_{1} u_{2}^{\prime} u^{+}-u^{-}\left(u^{+}\left(a r_{1}-q_{1} r_{2} / q_{2}\right)+q_{1} u_{2}^{\prime} p_{2} / q_{2}\right)} .
$$

By (4.17) and $u^{\prime}\left(\xi_{2}^{\varepsilon}\right) \rightarrow 0$, we immediately get $c_{2} \rightarrow 0$ as $\varepsilon \rightarrow 0^{+}$. Thus,

$$
c_{1} \rightarrow \frac{v^{-}}{u^{-}} \text {. }
$$

Returning to (4.5), by the uniform convergence of $u^{\varepsilon}(\xi)$ on $\left(-\infty, \xi_{0}-\delta\right)$ as well as $\xi_{\alpha}=\xi_{0}$, we get

$$
\left|p_{1}^{\varepsilon}(\xi)\right| \leq M \quad \text { for } \xi<\xi_{0}-\delta,
$$

where $M$ is independent from $\varepsilon>0$.

Thus, $\lim _{\varepsilon \rightarrow 0^{+}} v^{\varepsilon}(\xi)=v^{-}$uniformly for $\xi \in\left(-\infty, \xi_{0}-\delta\right)$. Similarly $\lim _{\varepsilon \rightarrow 0^{+}} v^{\varepsilon}(\xi)$ $=v^{+}$uniformly for $\xi \in\left(\xi_{0}+\delta,+\infty\right)$.

THEOREM 4.4. Suppose $U^{\varepsilon}(\xi)$ is a solution of (4.18) (constructed in (4.11)). Then $v^{\varepsilon}(\xi)-H_{1}\left(\xi-\xi_{0}^{\varepsilon}\right)$ converges weakly to $\bar{c} \cdot \delta(\xi-\sigma)$ as $\varepsilon \rightarrow 0^{+}$, where $\bar{c}=[u v]-\sigma[v]$,

$$
H_{1}(x)= \begin{cases}v^{-}, & x<0 \\ v^{+}, & x>0\end{cases}
$$

Theorem 4.4 can be obtained by the following steps.

(a) For a sloping test function $\phi \in C_{0}^{\infty}(R)$, from

$$
-\int_{R}(u-\xi) v^{\varepsilon} \phi^{\prime} d \xi+\int_{R} v^{\varepsilon} \phi d \xi=\varepsilon \int_{R} v^{\varepsilon} \phi^{\prime \prime} d \xi
$$


we immediately get

$$
\lim _{\varepsilon \rightarrow 0^{+}} \int_{R}\left(v^{\varepsilon}(\xi)-H_{1}\left(\xi-\xi_{0}^{\varepsilon}\right)\right) \phi(\xi) d \xi=([u v]-\sigma[v]) \phi(\sigma) .
$$

(b) From the weak maximum principle for the ordinary differential equation

$$
\left(\varepsilon v^{\prime}-(u-\xi) v\right)^{\prime}-v=0
$$

(which is similar to that in Gilbarg, Trudinger [5], Chapter 8, Exercise 8.1), we know

(i) $v^{+} \cdot v^{-}>0$ implies $v^{\varepsilon}(\xi) \geq 0$ (or $\left.\leq 0\right) \quad \forall \xi \in R$. Hence, $v^{\varepsilon}(\xi)-H_{1}\left(\xi-\xi_{0}^{\varepsilon}\right)$ is bounded from below (or above).

(ii) $v^{+} \cdot v^{-}<0$ implies $v^{\varepsilon}(\xi)$ possesses a unique null point $\xi_{3}^{\varepsilon}$ such that $v^{\varepsilon}\left(\xi_{3}^{\varepsilon}\right)=$ 0 and at most two critical points $\eta_{1}^{\varepsilon}, \eta_{2}^{\varepsilon}$ such that $v^{\prime}\left(\eta_{1}^{\varepsilon}\right)=v^{\prime}\left(\eta_{2}^{\varepsilon}\right)=0$.

(c) For case (i), employing the same approximation argument as in [11], we know that (4.22) holds for all $\phi \in C_{0}^{\infty}(R)$.

(d) For case (ii), $\lim _{\varepsilon \rightarrow 0^{+}} \xi_{3}^{\varepsilon}=\sigma$. Integrating (4.18) on $\left(-\infty, \xi_{3}^{\varepsilon}\right)$ and $\left(\xi_{3}^{\varepsilon},+\infty\right)$, respectively, we get

$$
\begin{gathered}
-\int_{-\infty}^{\xi_{3}^{\varepsilon}}(u-\xi) v^{\varepsilon} \phi^{\prime} d \xi+\int_{-\infty}^{\xi_{3}^{\varepsilon}} v^{\varepsilon} \phi d \xi=\varepsilon v^{\prime}\left(\xi_{3}^{\varepsilon}\right) \phi(\sigma)+\varepsilon \int_{-\infty}^{\xi_{3}^{\varepsilon}} v^{\varepsilon} \phi^{\prime \prime} d \xi \\
-\int_{\xi_{3}^{\varepsilon}}^{+\infty}(u-\xi) v^{\varepsilon} \phi^{\prime} d \xi+\int_{\xi_{3}^{\varepsilon}}^{+\infty} v^{\varepsilon} \phi d \xi=-\varepsilon v^{\prime}\left(\xi_{3}^{\varepsilon}\right) \phi(\sigma)+\varepsilon \int_{\xi_{3}^{\varepsilon}}^{-\infty} v^{\varepsilon} \phi^{\prime \prime} d \xi .
\end{gathered}
$$

For convenience, we assume $v^{-}>0>v^{+}$and $v^{\varepsilon}(\xi)$ possesses two critical points $\eta_{1}^{\varepsilon}, \eta_{2}^{\varepsilon}$ such that $\lim _{\varepsilon \rightarrow 0^{+}} \eta_{i}^{\varepsilon}=\sigma$. For the other cases, the following results can be obtained in a similar way.

Taking sloping test functions $\phi_{1}, \phi_{2} \in C_{0}^{\infty}(R)$ such that

$$
\phi_{1} \leq \phi \leq \phi_{2}, \quad\left|\phi(\sigma)-\phi_{i}(\sigma)\right| \leq \delta, \quad i=1,2 .
$$

Then

$$
\begin{gathered}
\int_{-\infty}^{\xi_{3}^{\varepsilon}}\left(v^{\varepsilon}(\xi)-H_{1}\left(\xi-\xi_{3}^{\varepsilon}\right)\right) \phi_{1} \leq \int_{-\infty}^{\xi_{3}^{\varepsilon}}\left(v^{\varepsilon}(\xi)-H_{1}\left(\xi-\xi_{3}^{\varepsilon}\right)\right) \phi \\
\leq \int_{-\infty}^{\xi_{3}^{\varepsilon}}\left(v^{\varepsilon}(\xi)-H_{1}\left(\xi-\xi_{3}^{\varepsilon}\right)\right) \phi_{2} \\
\int_{\xi_{3}^{\varepsilon}}^{+\infty}\left(v^{\varepsilon}(\xi)-H_{1}\left(\xi-\xi_{3}^{\varepsilon}\right)\right) \phi_{2} \leq \int_{\xi_{3}^{\varepsilon}}^{+\infty}\left(v^{\varepsilon}(\xi)-H_{1}\left(\xi-\xi_{3}^{\varepsilon}\right)\right) \phi \leq \int_{\xi_{3}^{\varepsilon}}^{+\infty}\left(v^{\varepsilon}(\xi)-H_{1}\left(\xi-\xi_{3}^{\varepsilon}\right)\right) \phi_{1} .
\end{gathered}
$$

Here we have ignored the integral on $\left[\xi_{5}, \xi_{6}\right]$ with $v\left(\xi_{5}\right)=v^{-}, v\left(\xi_{6}\right)=v^{+}$for $\left|\xi_{5}-\xi_{6}\right| \leq\left|\eta_{1}^{\varepsilon}-\eta_{2}^{\varepsilon}\right| \rightarrow 0$ and $\left|v^{\varepsilon}(\xi)\right| \leq \max \left(\left|v^{+}\right|,\left|v^{-}\right|\right)$on this interval.

From (4.23)-(4.26), we get

$$
\begin{aligned}
& -\lim _{\varepsilon \rightarrow 0^{+}} \varepsilon v^{\prime}\left(\xi_{3}^{\varepsilon}\right)\left(\phi_{2}(\sigma)-\phi_{1}(\sigma)\right)-\left(u^{-} v^{-}-\sigma v^{-}\right) \phi_{1}(\sigma)+\left(u^{+} v^{+}-\sigma v^{+}\right) \phi_{2}(\sigma) \\
& \quad \leq \int_{R}\left(v^{\varepsilon}(\xi)-H_{1}\left(\xi-\xi_{3}^{\varepsilon}\right)\right) \phi \\
& \quad \leq \lim _{\varepsilon \rightarrow 0^{+}} \varepsilon v^{\prime}\left(\xi_{3}^{\varepsilon}\right)\left(\phi_{2}(\sigma)-\phi_{1}(\sigma)\right)-\left(u^{-} v^{-}-\sigma v^{-}\right) \phi_{2}(\sigma)+\left(u^{+} v^{+}-\sigma v^{+}\right) \phi_{1}(\sigma) .
\end{aligned}
$$


Since $v^{\prime}\left(\xi_{3}^{\varepsilon}\right) \leq 0, \phi_{2}(\sigma)-\phi_{1}(\sigma)>0$, from (4.27), we know

$$
0 \geq \lim _{\varepsilon \rightarrow 0^{+}} \varepsilon v^{\prime}\left(\xi_{3}^{\varepsilon}\right) \geq-M_{1}>-\infty \text {. }
$$

Letting $\delta \rightarrow 0^{+}$, we get

$$
\lim _{\varepsilon \rightarrow 0^{+}} \int_{R}\left(v^{\varepsilon}(\xi)-H_{1}\left(\xi-\xi_{0}^{\varepsilon}\right)\right) \phi(\xi) d \xi=([u v]-\sigma[v]) \phi(\sigma) .
$$

Acknowledgment. I have received help from Professor T. Zhang and had an interesting discussion with Professor Li Zhou. Here I express my thanks to them.

\section{REFERENCES}

[1] C. M. Dafermos, Solution of the Riemann problem for a class of hyperbolic systems of conservation laws by the viscosity method, Arch. Rational Mech. Anal. 52, 1-9 (1973)

[2] C. M. Dafermos and R. J. DiPerna, The Riemann problem for certain classes of hyperbolic systems of conservation laws, J. Differential Equations 20, 90-114 (1976)

[3] P. Le Floch, Entropy weak solutions to nonlinear hyperbolic systems under nonconservative form, Comm. Partial Differential Equations 13 (6), 669-727 (1988)

[4] __, An existence and uniqueness result for two nonstrictly hyperbolic systems, Nonlinear Evolution Equations that Change Type (Keyfitz and Shearer, eds.), IMA series, vol. 27, Springer, New York, 1990, pp. 126-138

[5] D. Gilbarg and N. S. Trudinger, Elliptic Partial Differential Equations of Second Order, SpringerVerlag, Berlin and New York, 1977

[6] J. Glimm, Solutions in the large for nonlinear hyperbolic systems of equations, Comm. Pure Appl. Math. 18, 697-715 (1965)

[7] B. L. Keyfitz and H. C. Kranzer, A viscosity approximation to a system of conservation laws with no classical Riemann solution, Nonlinear Hyperbolic Problems, Lecture Notes in Math., no. 1402, Springer-Verlag, Berlin and New York, 1989, pp. 185-197

[8] D. J. Korchinski, Solution of a Riemann problem for a $2 \times 2$ system of conservation laws possessing no classical weak solution, Ph.D. Thesis, Adelphi University, Garden City, New York, 1977

[9] P. Lax, Hyperbolic systems of conservation laws. II, Comm. Pure Appl. Math. 10, 537-556 (1957)

[10] T.-P. Liu, The Riemann problem for general systems of conservation laws, J. Differential Equations 18, 218-234 (1975)

[11] D. C. Tan, T. Zhang, and Y. Z. Zheng, Delta-shock wave solutions for hyperbolic systems of conservation laws, submitted to J. Differential Equations

[12] A. I. Volpert, The space BV and quasilinear equations, Math. USSR-Sb. 2, 257-267 (1967) 